

\section{EQUAL BENEFITS}

One analysis has found that, compared with non-drinkers, both men and women who consume around one drink a day (the $>15$ grams group) have a lower risk of developing renal-cell cancer.

1.2

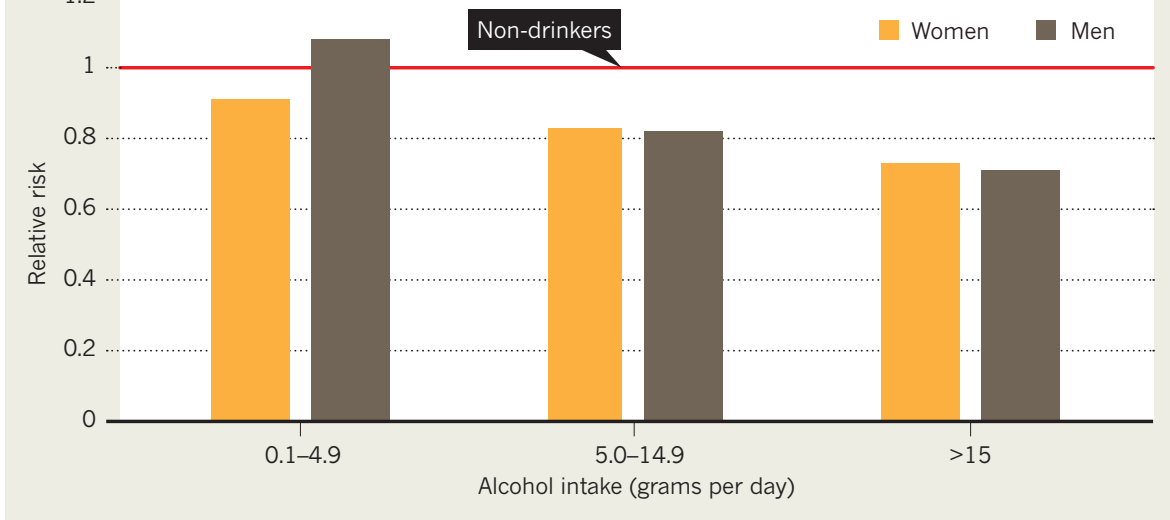

is broken up into molecules called reactive quinone species, which can damage DNA. Oestrogen levels are higher in women than in men, so if alcohol interacts with these molecules and stops them from damaging DNA, the reduction in kidney-cancer rates would be larger for women than for men.

To test this idea, Parker is investigating whether women who have genetic variations in the enzymes that metabolize alcohol also have differences in their kidney-cancer risk. Women who are genetically predisposed to metabolize alcohol quickly should get less protection from alcohol, because it is expelled faster. Parker expects the study to be published in the next six months.

\section{HOW MUCH IS ENOUGH?}

If alcohol is indeed protective, how much should you drink? Alicja Wolk, a nutritional epidemiologist at the Karolinska Institute in Stockholm, looked at about 59,000 Swedish women aged between 40 and 76 . Her study found that women who drink 2.5-4.3 grams a day, or 1-2 glasses of wine per week, were two-thirds as likely to develop kidney cancer as women who drank less than a glass a week ${ }^{5}$.

One question, Wolk says, is whether the beneficial effect of alcohol that seemed to disappear at higher dosages does so suddenly, tails off or simply flattens out. The first two wouldn't be unusual. "This occurs often in biology," she says. "For example, with vitamins you have an optimal amount and then it's too much." But there are few data to confirm or refute which is correct; not many of the women surveyed drank more than a few drinks per week.

Wolk found that women seemed to need to drink at least $2.5 \mathrm{~g}$ of alcohol per day to gain protection compared with those who drank less. Women who drank more than $4.3 \mathrm{~g}$ per day were not protected any more than those who drank less than a glass of wine a week.

One confounding factor, Wolk notes, is that there are often differences in alcohol consumption between men and women.
Swedish middle-aged women see drinking as less acceptable than their male counterparts. Younger Swedish women drink more, she says, but the study didn't include these younger cohorts. Wolk's study used self-reported data and did not draw a distinction between episodic drinking and everyday moderation. To determine whether drinking behaviour makes a difference would require a more sensitive measure of intake.

Wolk says that she can only speculate about what the biological mechanism might be. One potential clue is that alcohol seems to have more of a protective effect in overweight women. This might point to a link between alcohol and triglycerides, cholesterol, lowdensity lipoproteins and insulin. Previously, postmenopausal women who drank have been found to have decreased levels of each.

\section{Parker adds that}

there is a possibility that the alcohol is interacting in some way with other substances in the diet, or that the type of alcoholic beverage might be a factor. Such effects are difficult to tease out, he says. Lee's 2012 meta-analysis showed no significant differences in the protective effects of beer, wine or spirits.

The situation is further complicated because kidney cancer is not one, but several diseases. Clear-cell carcinoma is the most common form, making up some $70 \%$ of cases. Few data exist on the rarer types such as papillary renalcell carcinoma. Parker adds that the different cancers are heterogeneous enough that the protective effect of alcohol might not apply to all of them, and that case-control and cohort studies are difficult to do because of the rarity of some types of kidney cancer. There were about 62,700 cases of all types of kidney cancer in the United States in 2015. The papillarycell type represents only about 1 in 10 of these; other cancers are even less common.
Lee notes that kidney-cancer studies have been done mainly in Europe and North America. Although US studies include some African Americans, it is not clear whether the findings apply to the global population. "People think, will this apply to Middle Easterners? Asians? We need a larger consortium or study that has also African Americans, or Asians, or East Asians," she says.

It is also important to separate the effects of different risk factors. Lee noted that her 2007 study showed that alcohol retained its protective effect even among smokers, people with a high body mass index and those with hypertension. But to check different combinations of risk factors (for example, smoking and obesity compared with smoking alone) would require a different statistical method. Finding funding for in-depth epidemiological studies of kidney cancer can be difficult, however, because the disease is not as common as other cancers, and not as deadly as gliomas, or liver or pancreatic cancer. "Kidney cancer is in this kind of limbo," says Parker.

Lee is hoping to do further work in countries such as South Korea to reveal any differences between ethnic groups, as well as to study more women who drink higher amounts of alcohol, to investigate whether the possible difference between genders holds up for heavier drinkers.

Parker, meanwhile, is optimistic that future studies of the genetics of cancer may yield some insights. "That's where we move next," he says. "Alcohol seems to be protective, but maybe it's against a certain molecular type. Environment is another factor; maybe there's some gene-environment interaction."

It's entirely possible that alcohol in combination with some other lifestyle factor is the key. Parker draws a parallel with resveratrol, the once-touted protector of heart disease. The problem with the initial findings was that there could have been many co-factors involved in red-wine consumption. "Maybe they were so chilled out they haven't got a heart-disease problem," he says. In that case, the red wine might have correlated with happiness, or habits that generally reduced stress. Perhaps there was some interaction with the foods that go with red wine. The question for kidney cancers is similar - is the tipple protective, or is it just the good meals and good times that go with it?

Jesse Emspak is a freelance science writer based in New York City.

1. Lee, J. E. et al. J. Natl Cancer Inst. 99, 801-810 (2007).

2. Song, D. Y., Song, S., Song, Y. \& Lee, J. E. Br. J. Cancer 106, 1881-1890 (2012).

3. Parker, A. S., Cerhan, J. R., Lynch, C. F., Ershow, A. G. \& Cantor, K. P. Am. J. Epidemiol. 155, 455-462 (2002).

4. Cheng, G. \& Xie, L. Arch. Med. Sci. 7, 648-657 (2011).

5. Rashidkhani, B., Akesson, A., Lindblad, P. \& Wolk, A. Int. J. Cancer 117, 848-853 (2005). 